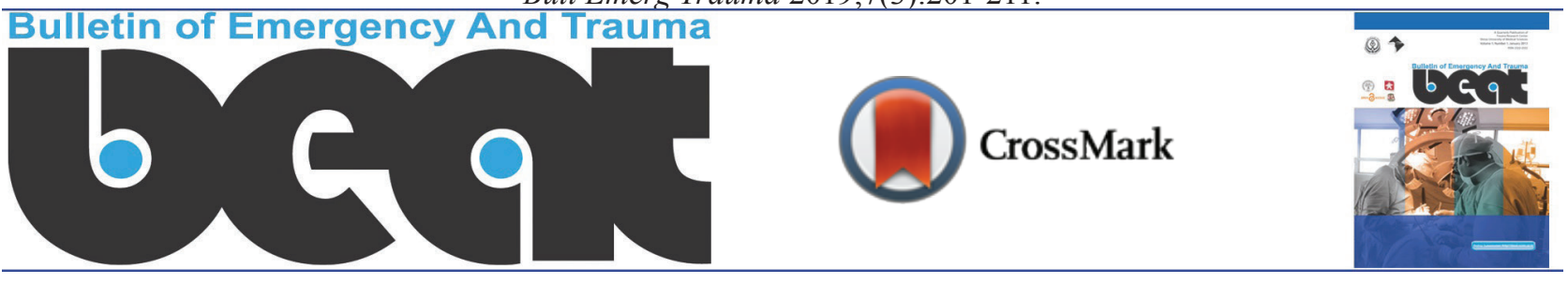

\title{
Assessing Preparedness of Non-Hospital Health Centers to Provide Primary Emergency Care; A Systematic Review
}

\author{
Mehrdad Amir Behghadami ${ }^{1,2,3}$, Ali Janati $^{1 *}$, Homayoun Sadeghi-Bazargani ${ }^{3}$, Masoumeh Gholizadeh $^{1}$, Farzad \\ Rahmani $^{4,3}$, Morteza Arab-Zozani ${ }^{1}$
}

\begin{abstract}
${ }^{1}$ Iranian Center of Excellence in Health Management (IceHM), Department of Health Service Management, School of Management and Medical Informatics, Tabriz University of Medical Sciences, Tabriz, Iran

${ }^{2}$ Student Research Committee (SRC), Tabriz University of Medical Sciences, Tabriz, Iran

${ }^{3}$ Road Traffic Injury Research Center, Tabriz University of Medical Sciences, Tabriz, Iran

${ }^{4}$ Emergency Medicine Department, Sina Medical Research and Training Hospital, Tabriz University of Medical Sciences, Tabriz, Iran
\end{abstract}

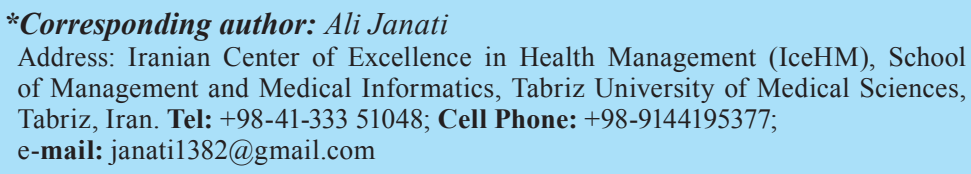

Received: January 12, 2019

Revised: May 21, 2019

Accepted: May 31, 2019

\section{ABSTRACT}

Objectives: To identify prevalent domains related to the concept of assessing preparedness of non-hospital centers to provide primary emergency care in order to develop a comprehensive framework.

Methods: Five databases including PubMed, Scopus, Web of science, Barakat Knowledge Network Systems (BKNS) and Scientific Information Database (SID) were searched in English and/or Persian languages with no time limit until March, 2018. Manual search and grey literature were also done. According to the eligibility criteria, all the studies were independently tracked by two researchers. Studies were appraised using the Mixed Methods Appraisal Tool (MMAT). The findings were synthesized through directed content analysis method. Results: Out of 3014 studies, 15 studies were included for data synthesis. The synthesis of literature resulted in the emergence of 13 domains and 25 sub-domains. Then, they were categorized based on Donabedian's triple model and a conceptual framework was developed. In this framework, 6 domains were put in input, 6 in processes, and 1 domain in outcome. Of the 15 included studies, 1 study considered 10 domains and 14 other studies considered 4 to 8 domains out of 13 synthesized domains. The most prevalent synthesized domains were "medical supplies and equipment" and "human resources", which were considered in 15 studies.

Conclusion: In this study, a conceptual framework was constructed that identifies elements that significantly affect the preparedness of these centers. This framework may assist managers to take a comprehensive approach to assess these centers.

Keywords: Non-hospital health centers; Primary emergency care; Preparedness; Assess.

Please cite this paper as:

Behghadami MA, Janati A, Sadeghi-Bazargani H, Gholizadeh M, Rahmani F, Arab-Zozani M. Assessing Preparedness of Non-Hospital Health Centers to Provide Primary Emergency Care; A Systematic Review. Bull Emerg Trauma. 2019;7(3):201-211. doi: 10.29252/beat-070301. 


\section{Introduction}

$\mathrm{N}$ on-hospital centers include rural health centers, which are the only government medical units with a nearby predicated health house in rural areas, and urban primary health care centers, health complexes, and outpatient clinics $[1,2]$. These centers are considered to be social organizations and the most important centers of health care system in society [3]. Along with hospitals, Emergency Medical Service (EMS), and other specialist outpatient clinics and polyclinics, cooperation of these centers to provide emergency care is an advantage which supplies the highest number of potential services to the highest possible number of individuals [4].

Diseases resulting from sudden attacks and time sensitive diseases are the most critical concerns of common health. Particularly, effects of these diseases in countries with low to moderate income are so severe that $45 \%$ of death rate and $36 \%$ of disease burden is for such diseases which require emergency care. It seems that improving access to high quality emergency care in these countries can lead to decrease in death rate by 21 million and disability-adjusted life years by 501 individuals [5]. As all life threatening hazards always occur suddenly and unexpectedly [4], it seems essential that this unit be responsible for providing individuals with emergency care under any conditions, ranging from delays in the arrival of ambulances to other extraordinary conditions [6, 7]. Moreover, it should have the required preparation and optimally provide Basic Life Support (BLS) by means of organized infrastructures such as physical space, medical equipment, emergency medicines, support facilities, and human forces with high clinical-medical knowledge and skills [1].

Providing primary emergency care at non-hospital health centers' has been recommended by a variety of studies as a strategy to reduce burden of hospitals' emergency ward $[1,4,8,9]$. But, the point is if these non-hospital centers have been decently organized to provide emergency care or not. A number of international studies have consistently reported the lack of these centers' preparedness in terms of emergency medicines, equipment, and support facilities [7, 10-12]. It appears that non-hospital health care centers may arrive at their goals only if they have accurate information about current state. Hence, existence of an efficient and effective assessment system can play a pivotal role in realizing the capabilities of such centers and providing patients with optimal emergency care [13].

Up to now, there have been few studies on this issue in Iran. Only some of these studies have used checklists to probe into gaps and potential weaknesses $[1,4,7]$. Based on our investigation, no standard and valid tool has been designed to assess preparedness of non-hospital health centers for offering primary emergency care. Also, there is no agreement as to a standard, comprehensive, reliable tool which could be used to assess preparedness of non-hospital health centers for providing primary emergency care. Therefore, a comprehensive framework is required to assess preparedness of non-hospital health centers. The purpose of the present systematic review is to identify prevalent domains related to the concept of assessing preparedness of non-hospital centers to provide primary emergency care in order to develop a comprehensive framework.

\section{Materials and Methods}

\section{Protocol and registration}

A systematic review was conducted according to the Preferred Reporting Items for Systematic Reviews and Meta-Analyses (PRISMA) [14]. The review protocol was registered in PROSPERO (registration no. CRD42018096044; http://www. crd.york.ac.uk/PROSPERO/display_record. php?ID=CRD42018096044).

\section{Data sources and Search Strategy}

A comprehensive search strategy was devised to identify studies which focused on the non-hospital health centers and emergency care. We searched five databases including PubMed, Scopus, Web of science, Barakat Knowledge Network System, and Science Information Database (SID). The searches were performed in English and/or Persian languages with no time limit until March, 2018. It is to notify that SID, which was established in August 2004, is a database which classifies Iranian science and research journals in science groups (http://www. sid.ir/En/Journal/) [15]. Also, Barakat knowledge network system (BKNS) was established in 2012 to cover seven science fields. Health sector is the first phase of this system. Indeed, through developing a comprehensive database of the articles taken from health journals and conferences, this part of the system has turned Iran into information corridor of Islamic world (http://health.barakatkns.com) [16].

A combination of MeSH term and free words (words selected by the research team) were used to maximize research sensitivity. English key words and their Persian equivalents were used. English key words included: "Health Center" ,"Primary Care Center" ,"Primary Health Care Center" ,"Health Post" ,"Health House" ,"Rural Health Center," "Urban Health Center" ,"Health Care Center," "Outpatient Clinic" ,"Ambulatory Health Center", Non-hospital, Emergency, "Emergency Services«, "Emergency Care", "Emergency Medical Services", "Emergency Case", "Critical Emergency", 'Basic Life Support", "Basic Emergency Care", "Basic Emergency Services", "Primary Emergency Care", "Primary Emergency Services", Trauma, Pre hospital, Preparedness, Ready, Readiness, Assess, Evaluation, Appraisal, "Performance Evaluation", "Performance Appraisal", "Quality Assessment", 
Inspection.

The search syntax on PubMed:

1. "Health Center" [MeSH Term]

2. "Primary?Care Center" ti,ab.

3. "Primary Health?Care Center" ti,ab.

4. "Health Post" ti,ab.

5. "Health House" ti,ab.

6. "Rural Health Center" ti,ab.

7. "Urban Health Center" ti,ab.

8. "Health Care Center" ti,ab.

9. "Out?patient Clinic" ti,ab.

10. "Ambulatory Health Center" ti,ab.

11. Non?hospital ti,ab.

12. Emergency ti,ab.

13. "Emergency Services" ti,ab.

14. "Emergency Care" ti,ab.

15. "Emergency Case" ti,ab.

16. "Critical Emergency" ti,ab.

17. "Basic Life Support" ti,ab.

18. "Basic Emergency Care" ti,ab.

19. "Basic Emergency Services" ti,ab.

20. "Primary Emergency Care" ti,ab.

21. "Primary Emergency Services" ti,ab.

22. Trauma ti,ab.

23. Pre?hospital ti,ab.

24. Prepar* ti,ab.

25. Ready ti,ab.

26. Readiness ti,ab.

27. Asses* ti,ab.

28. Evaluat* ti,ab.

29. Apprais* ti,ab.

30. "Performance Evaluation" ti,ab.

31. "Performance Appraisal" ti,ab.

32. "Quality Assessment" ti,ab.

33. Inspection ti,ab.

34. 1 Or 2 Or 3 Or 4 Or 5 Or 6 Or 7 Or 8 Or 9 Or 10 Or 11

35. 12 Or 13 Or 14 Or 15 Or 16 Or 17 Or 18 Or 19 Or 20 Or 21 Or 22 Or 23

36. 24 Or 25 Or 26 Or 27 Or 28 Or 29 Or 30 Or 31 Or 32 Or 33

\section{34 AND 35 AND 36 AND}

In order to identify the other studies, which were not identified in databases search, a manual search was done on reference list, citation of the included studies, and searching ResearchGate social network. In addition, a grey literature of institutions and organizations (for instance, health ministry, treatment and health vice chancellery, East Azerbaijan Province) and other organizations that publish studies (like WHO) was performed to identify other related studies such as reports and etc.

\section{Selection Criteria}

Studies were expected to meet all of these criteria.

\section{Inclusion criteria:}

- Studies related to Primary Health Care Centers (PHCCs) or outpatient clinics.

- Studies that reported at least one domain and item related to the preparedness in providing primary emergency care.

- English and/or Persian articles.

\section{Exclusion criteria:}

- Studies conducted in emergency wards of hospitals, outpatient special clinics affiliated with hospitals and EMSs were excluded from the study since offering emergency care is one of their main responsibilities. Hence, they should be completely prepared to deal with emergent cases.

- Studies associated with preparedness in human and natural disasters such as flooding, earthquakes, battles, and etc.

- Articles whose full text is not available.

- Non-English articles

- Non-quality studies (studies that have not met the two primary questions of the MTMT tool.)

\section{Study Selection}

All the records were imported into EndNote software package (V.X6). The duplicate records were deleted. According to the inclusion criteria, all the titles, abstracts and full text were independently screened by two researchers (authors land 4). Then, the disagreements between the researchers were resolved through reaching a unanimous view. If there was a disagreement, it was solved through consulting a third researcher (author 2).

\section{Ouality Assessment}

Quality appraisal of the included studies was independently performed by two researchers (authors 1and 4). Any disagreements were solved through discussions or exchanging views and if required through a decision made by a third researcher (author 2). Quality methodology of all the included studies was appraised by means of Mixed Methods Appraisal Tool [17]. Validity and reliability of the tool were approved and the accepted standards were met $[18,19]$. At present, MMAT is the best and most comprehensive tool available for appraising multi-method studies. MMAT is used to simultaneously investigate all three studies of methodologies, including mixed methods, qualitative, and quantitative (randomized controlled, nonrandomized, and descriptive). The total score of the methodology was calculated in percentage. The criteria used to determine quality score based on the design of the study are different [17].

For each of the included articles, total quality score may not contain useful data (in comparison with descriptive summary through using MMAT criterion); however, it is possible to calculate it by means of MMAT. Since there are only a few criteria for each domain, the score can be represented through descriptors like *,**, ***, and ****. For quantitative and qualitative studies, this score can be the number of the criteria met, which is divided into four (categories from $25 \%\left(^{*}\right)$-one criterion 
met- to $100 \%(* * * *)$-all the criteria met-). For mixed methods design studies, the hypothesis is that the total quality of a combination cannot exceed the quality of its weakest component. Thus, the total quality score is the lowest score of the study components. The score is $25 \%\left(^{*}\right)$ when QUAL $=1$ or QUAN $=1$ or $\mathrm{MM}=0$; it is $50 \%(* *)$ when $\mathrm{QUAL}=2$ or $\mathrm{QUAN}=2$ or $\mathrm{MM}=1$; it is $75 \%(* * *)$ when $\mathrm{QUAL}=3$ or $\mathrm{QUAN}=3$ or $\mathrm{MM}=2$; and it is $100 \%(* * * *)$ when $\mathrm{QUAL}=4$ and QUAN=4 and $\mathrm{MM}=3$ (QUAL being the score of the qualitative component; QUAN the score of the quantitative component; and MM the score of the mixed methods component). (Supplementary File 1) [17].

\section{Data Extraction}

Based on the purpose of the study, for each stage of the extraction procedure, recording and analyzing data, data extraction forms and tables were designed by the researchers. The main characteristics of the included studies and their respective results were summarized based on the following data: purpose of the study, type of the centers, publication year of the studies, MMAT score, and type of the studies (quantitative, qualitative, mixed, and etc.).

\section{Data Synthesis and Analysis}

Content and provided information of the included studies were synthesized through directed content analysis method $[20,21]$ and were categorized based on Donabedian model [22]. It included coding the identified items of the studies into domains and subdomains, which was carried out through deductive reasoning [21]. Directed content analysis is conducted through a structured approach which resorts to the existing theory (or research) in order to identify key concepts as a way to categorize primary codes [21]. Primary categorization of recommended codes was identified by means of available research and guidelines concerning preparedness of nonhospital centers to provide primary emergency care. The process of collecting and analyzing qualitative data was conducted by the first researcher (author 1) while the second researcher (author 4) was consistently consulted in relation to coding and the emerging domains. Any disagreement was resolved through consultation with the third researcher (author 2). Donabedian model is used as a basis for defining quality. "Structure" stands for features of the health units and centers where care is provided. It includes such features as financial resources, human resources, equipment, and etc. "Process" points to any activity related to providing services, interaction between customers, and structure of health care. "Outcome" indicates effects and consequences of any given care on health condition. Structure affects processes and outcomes. Outcome reflects the effects of structure and process combined [22].

\section{Results}

\section{Study Selection}

Out of 3014 retrieved studies, 15 studies were included for data synthesis. (Figure 1)

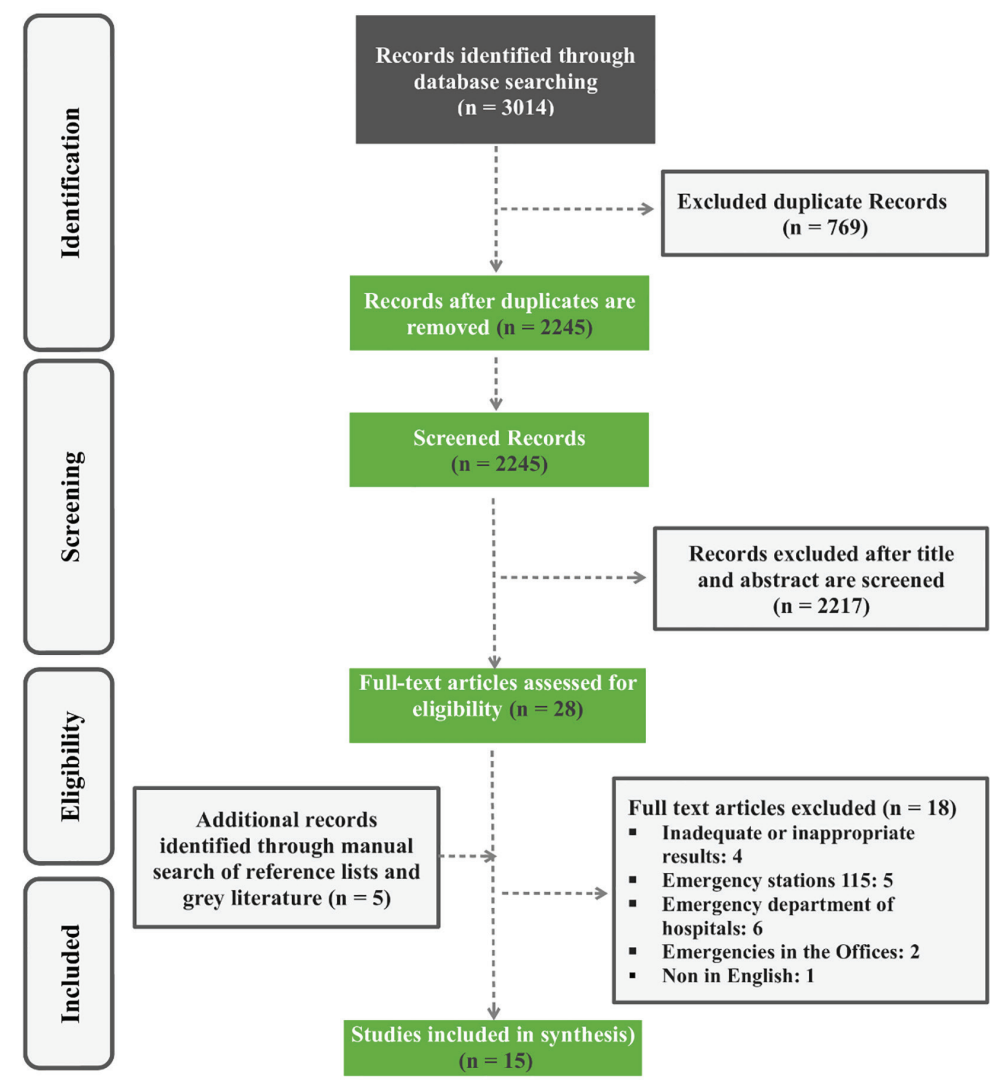

Fig. 1. Review selection process and results, in accordance with the PRISMA guidelines. 


\section{Study Characteristics}

The included studies were published between 2004 and 2018. Out of these studies 4 and 11 studies were published from 2004 to 2007 and from 2008 to 2018 , respectively. It could be inferred that publication of such studies in this field has remarkably increased over recent decades. Such studies have been generally conducted in developing countries. Study method of 12 studies out of the 15 included studies was quantitative, 2 were mixed methods and one was not applied. 14 studies out of the 15 included studies were published in English and only one study was in Persian. 10 out of the 15 included studies were on preparedness of PHCCs, 3 were on preparedness of outpatient clinics, and 2 were about preparedness of both of these centers, which provide primary emergency care. (Table 1)

\section{Ouality Assessment}

2 of the studies were ranked as low quality $(\mathrm{MMAT}=25 \%), \quad 6$ as medium qualities (MMAT $=50 \%$ ), and 6 as acceptable qualities (MMAT=75\%). There was no study to be ranked as weak quality (MMAT $=0 \%$ ) or very high quality (MMAT $=100 \%$ ). MMAT tool was not applied in one of the studies. (Table 1)

\section{Synthesis of Results}

Our findings indicated 13 domains and 25 subdomains of the included studies (Table 2). A conceptual framework was presented. In this conceptual framework, 6 domains were put in inputs, 6

Table 1. Main characteristics of included studies

\begin{tabular}{|c|c|c|c|c|}
\hline Authors & $\begin{array}{l}\text { Center } \\
\text { types }\end{array}$ & Purposes & Study types & $\begin{array}{l}\text { MMAT } \\
\text { scores }\end{array}$ \\
\hline $\begin{array}{l}\text { Aloufi et al } \\
2016\end{array}$ & PHCCs & $\begin{array}{l}\text { Estimating prevalence of emergency cases in PHCCsand investigating } \\
\text { barriers physicians face in PHCCswhen dealing with such emergency cases }\end{array}$ & $\begin{array}{l}\text { Quantitative / } \\
\text { Cross-sectional }\end{array}$ & $25 \%$ \\
\hline $\begin{array}{l}\text { Yaman et al } \\
2008\end{array}$ & PHCCs & $\begin{array}{l}\text { Evaluating accessablity of emergency equipement and staff's level of } \\
\text { knowledg at PHCCs }\end{array}$ & $\begin{array}{l}\text { Quantitative / } \\
\text { survey }\end{array}$ & $25 \%$ \\
\hline $\begin{array}{l}\text { Nakahara et } \\
\text { al } 2009\end{array}$ & PHCCs & $\begin{array}{l}\text { Evaluating available resources for trauma care in health centers and refereal } \\
\text { hospitals of rural areas in Cambodia }\end{array}$ & $\begin{array}{l}\text { Quantitative / } \\
\text { Cross-sectional }\end{array}$ & $75 \%$ \\
\hline $\begin{array}{l}\text { Alsaad et al } \\
2017\end{array}$ & PHCCs & $\begin{array}{l}\text { Evaluating accessability of human and nonhuman resources for emergency } \\
\text { medical services at PHCCs }\end{array}$ & $\begin{array}{l}\text { Quantitative / } \\
\text { Cross-sectional }\end{array}$ & $75 \%$ \\
\hline $\begin{array}{l}\text { Razzak et al } \\
2008\end{array}$ & $\begin{array}{l}\text { PHCCs } \\
\text { (BHUs \& } \\
\text { RHCs) }\end{array}$ & $\begin{array}{l}\text { Assessment of the availability and quality of facility-based emergency } \\
\text { medical care }\end{array}$ & $\begin{array}{l}\text { Quantitative / } \\
\text { Cross-sectional }\end{array}$ & $50 \%$ \\
\hline $\begin{array}{l}\text { Razzak et al } \\
2013\end{array}$ & $\begin{array}{l}\text { PHCCs } \\
\text { (BHUs \& } \\
\text { RHCs) }\end{array}$ & $\begin{array}{l}\text { Evaluating emergency and trauma care facilities in four districts of the } \\
\text { province of Sindh, Pakistan }\end{array}$ & $\begin{array}{l}\text { Quantitative / } \\
\text { Cross-sectional }\end{array}$ & $50 \%$ \\
\hline $\begin{array}{l}\text { Nelson et al } \\
2015\end{array}$ & Clinics & $\begin{array}{l}\text { Better understaning of needs and capacities of island's emergency health } \\
\text { care and determing how emergency care can be improved among the } \\
\text { island's population }\end{array}$ & Mixed methods & $50 \%$ \\
\hline $\begin{array}{l}\text { Hsia et al } \\
2011\end{array}$ & PHCCs & $\begin{array}{l}\text { Evaluating facility capacities of health care in some subsaharan African } \\
\text { countries to expand accessablity of emergency care }\end{array}$ & $\begin{array}{l}\text { Quantitative / } \\
\text { survey }\end{array}$ & $75 \%$ \\
\hline $\begin{array}{l}\text { Burke et al } \\
2014\end{array}$ & $\begin{array}{l}\text { PHCCs/ } \\
\text { Clinics }\end{array}$ & $\begin{array}{l}\text { An assessment of emergency and urgent healthcare capabilities across all } \\
\text { levels of facilities in Kisumu and Siaya counties of western Kenya }\end{array}$ & $\begin{array}{l}\text { Quantitative / } \\
\text { survey }\end{array}$ & $75 \%$ \\
\hline $\begin{array}{l}\text { Arreola- } \\
\text { Risa et al } \\
2006\end{array}$ & Clinics & $\begin{array}{l}\text { Identifying affordable, sustainable methods to strengthen trauma care } \\
\text { capabilities in Mexico }\end{array}$ & $\begin{array}{l}\text { Quantitative / } \\
\text { survey }\end{array}$ & $50 \%$ \\
\hline $\begin{array}{l}\text { Mohey et al } \\
2017\end{array}$ & PHCCs & $\begin{array}{l}\text { 1. evaluating PHCC's structure for providing emergency care. } \\
\text { 2. evaluating view and function of service providers in PHCCs toward } \\
\text { emergecny care. } \\
\text { 3. identifying felt needs of service providers to further their medical studies } \\
\text { on emergency care. } \\
\text { 4. assessing the patterns for using emergency services in PHCCs } \\
\text { 5. evaluating customers satisfaction level of emergency services in PHCCs }\end{array}$ & Mixed methods & $75 \%$ \\
\hline $\begin{array}{l}\text { Mahfouz et } \\
\text { al } 2007\end{array}$ & PHCCs & $\begin{array}{l}\text { Evaluating emergency services at PHC level in terms of structure and } \\
\text { outcome, considering knowledge, view, and function of PHC physicians( } \\
\text { service providers), identifying their needs for furthering their medical } \\
\text { training in emergency care, and considering satisfaction with and pattern } \\
\text { of using emergency services from the view point of those who buy PHCCs' } \\
\text { services (customers) }\end{array}$ & $\begin{array}{l}\text { Quantitative / } \\
\text { Cross-sectional }\end{array}$ & $50 \%$ \\
\hline $\begin{array}{l}\text { Heidari et al } \\
2016\end{array}$ & PHCCs & $\begin{array}{l}\text { Investigating human and physical resources of health houses and health } \\
\text { centers to provide emergency services }\end{array}$ & $\begin{array}{l}\text { Quantitative / } \\
\text { Cross-sectional }\end{array}$ & $50 \%$ \\
\hline $\begin{array}{l}\text { Mock et al } \\
2006\end{array}$ & Clinics & To strengthen trauma care capabilities in four countries & $\begin{array}{l}\text { Quantitative / } \\
\text { survey }\end{array}$ & $50 \%$ \\
\hline $\begin{array}{l}\text { Mock et al } \\
2004\end{array}$ & $\begin{array}{l}\text { PHCCs/ } \\
\text { Clinics }\end{array}$ & Guidelines for essential trauma care & Not applicable & $\begin{array}{l}\text { Not ap- } \\
\text { plicable }\end{array}$ \\
\hline
\end{tabular}


Table 2. Domains, Sub-domains and Items Emerging From the Included Studies

Domains

Inputs

Medical supplies and equipment

\section{Sub-domains and items}

For Resuscitation ( Airway, Breathing and Circulation ): e.g., side lamp with stand,

dressing trays, dressing table, urinary catheter, forceps, Magill forceps, scissors, suture materials, needle holder, suction apparatus, blades, IV stand, nasogastric tubes, cannulas and urinary catheters, needle syringe, Cervical collars, oxygen mask, oxygen cylinder with Standard fitting, Bag-valve-mask, Nebulizer, trolley, tracheostomy sets, intravenous fluid Administration Sets, Nasal prongs, chest tube, oral or nasal airway, gauze and bandage, Arterial tourniquet

- $\quad$ For specific injuries: e.g. Pocket torch, Glasgow Coma Scale sheet, Reflex hammer, Sling, Splint, Cervical collars, Backboard, Sandbag

- $\quad$ Supplies safety for health care personnel: e.g., gloves, goggles and etc

Support facilities

- Diagnostic equipment : e.g. X-ray, Equipped ambulance cars, Stethoscope, Sphygmomanometer, Electrocardiograph, Minor Surgical Set, Cardiac Monitor, Defibrillator, Blood pressure cuff, Glucometer, Ultrasoundor, Otoscope, Ophthalmoscope, Laryngoscope, oesophageal detector device, Thermometer, Foetal stethoscope, Paediatric length-based (Broselow) tape,

- $\quad$ Sterilization equipment: e.g. Autoclave, hot-air oven, dry heat or four

- $\quad$ Physical space: presence of devoted place for emergency services and/or minor surgeries, location in the center (e.g., ground floor, near the entrance, separate entry, presence of sloped entry for the trolley, etc.), Separate drug cabinets for emergency services, Devoted registry for emergency case.

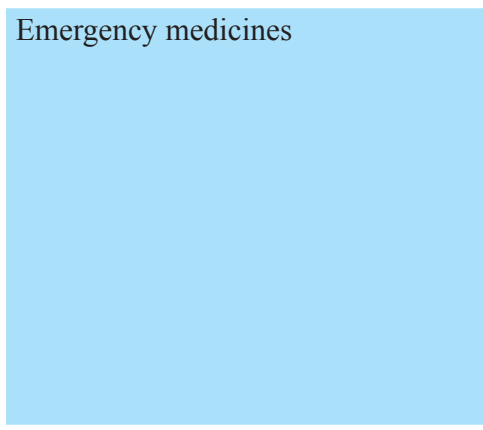

Human resources Anaesthesia (lidocaine, oxygen, diazepam, atropine), Pain, fever, inflammation (morphine, codeine, acetylsalicyclic acid, ibuprofen \& paracetamol), Anaphylaxis (dexamethasone, hydrocortisone \& epinephrine), Poisoning (naloxone), Anticonvulsants (phenobarbital, phenytoin \& magnesium sulphate), Infections (amoxycillin/ampicillin, amoxycillin \& clavulanic acid, benzylpenicillin, chloramphenicol, ciprofloxacin, cloxacillin, gentamicin, metronidazole, sulfamethoxazole \& trimethoprim), Diuretics (furosemide \& mannitol), Fluid and electrolyte balance (glucose solution $(5 \%, 50 \%)$, normal saline solution ( $0.9 \%$ isotonic), glucose with sodium chloride $(4 \%$ glucose, $0.18 \% \mathrm{NaCl})$, Ringer's lactate solution, potassium chloride solution) Calcium chloride injection, antihistaminic injection, activated charcoal powder, metoclopramide, furosemide, hyoscine, Bronchodilators, Antiscorpion\& snake(Antivenin), Antihypertensive Drugs (oral), Prednisolone, Met ergotamine

- $\quad$ List of emergency room staff (physicians and nurses) who have been trained in first aid and cardiopulmonary resuscitation (CPR)

- $\quad$ The number of personnel and ratio

Protocol \& Guideline

Proscess

- Clinical: e.g., Treatment protocol in early management of acute coronary syndrome and treatment of multi-trauma patients, Written clinical practice guidelines for providing primary emergency care, Guidelines for pediatric emergency triage, assessment and treatment (ETAT), protocols for the refered patients

- $\quad$ Non-clinical: e.g., Protocols for appropriate sterilization techniques

Assessment of the resuscitation and specific injuries processes

Asse

Staff Knowledge and skills (medical, nursing and others) in resuscitation: eg, 1) Assessment of airway compromise 2)manual manoeuvres (chin lift, jaw thrust, recovery position, etc.) 3)Insertion of oral or nasal airway 4)Assisted ventilation using bag-valvemask 5)Endotracheal intubation 6)Use of suction 7)Cricothyroidotomy (with or without tracheostomy) 8) Administration of oxygen 9)Needle thoracostomy 10)Three-way dressing 11)Assessment of shock 12)Compression for control of haemorrhage 13)Arterial tourniquet in extreme situations 14)Splinting of fractures for haemorrhage control 15)Pelvic wrap for haemorrhage control 16)Reading ECG 17)Nebulisation \& oxygen therapy 18)Simple suturing 19)NGT insertion 20)Administering IV fluid \&medications 21)Inserting IV cannula 22)Cardiac compression 23)Defibrillation

- $\quad$ Staff Knowledge and skills (medical, nursing and others) in specific injuries: eg, 1) Recognize altered consciousness; lateralizing signs, pupils 2)External pressure for bleeding 3)Packing, balloon tamponade for bleeding 4)Adequate pain control for chest injuries/ rib fractures 5)Clinical assessment of abdominal injuries (Visual examination, Percussion, Palpation \&Auscultation) 6)Immobilization: using two sandbags for spinal injuries 7)Logroll/log-lift 8)Assessment - recognition of presence or risk of spinal injury 9)Immobilization: C-collar, back board 10)Recognition of neurovascular compromise; disability-prone injuries

11)Basic immobilization (sling, splint) 12)Assessment of depth and extent burns and wounds 13)Sterile dressings 14) Use Autoclave and four

$\begin{array}{ll}\text { Maintenance of equipment } & \text { System for maintenance and repair of building, infrastructure \& equipment } \\ \text { equipment and appraise basic knowledge of the staff on calibration procedure of equipemtn. }\end{array}$


Medicine storage capability

\begin{tabular}{|c|c|}
\hline Infection control & $\begin{array}{l}\text { Availability of infection control items: e.g., soap, running water, sharp box, latex } \\
\text { gloves and disinfectants in assessed areas } \\
\text { - } \\
\text { waste e.g., burning and dumping, service location or waste disposal area without infectious } \\
\text { waste }\end{array}$ \\
\hline Educating and training & $\begin{array}{l}\text { Training courses for basic emergency management: e.g., BLS, Advanced Cardiac } \\
\text { Life Support (ACLS), Advanced Truma Life Support (ATLS) and Pediatric Advanced Life } \\
\text { Support courses, Newborn Resuscitation Program } \\
\text { Continuing Medical Education: two criteria are used appraise continuous training: } \\
\text { 1. At least } 50 \% \text { of service providers should have taken in-service training courses over last } 12 \\
\text { months. } 2 \text {. Over last } 6 \text { months, at least } 50 \% \text { of service providers have reported their personal } \\
\text { observations on this case }\end{array}$ \\
\hline Quality control & $\begin{array}{l}\text { Management capability: e.g., management committee meetings at least every } 6 \\
\text { months and recent documents of these meetings should be appraised } \\
\text { related documentation } \\
\text { Q Referral systems: observation of referral notes or patient records, Number of } \\
\text { emergency cases referred to the district hospital }\end{array}$ \\
\hline $\begin{array}{l}\text { Outcome } \\
\text { Patients' satisfaction }\end{array}$ & $\begin{array}{l}\text { Using these components e.g. accessibility of care, availability, inter-personal } \\
\text { relationship, informing patients, continuity of care, effectiveness of emergency care and } \\
\text { timely care }\end{array}$ \\
\hline
\end{tabular}

in processes, and 1 domain in outcome. The processes themselves are categorized into three processes of service, support, and management. Processes related to resuscitation are considered as service processes. Equipment and infrastructures maintenance, emergency medicines storage, infection control, and quality control are considered to be support processes. While, training and education are management processes (Figure 2). Out of the 15 included studies, one study [23] considered 10 synthesized domains and 14 other studies $[1,4,5,7,24-33]$ considered 4-8 domains of all the 13 synthesized domains. In these studies, the most prevalent synthesized domains were "medical supplies and equipment" and "human resources", which were considered in 15 studies $[1,4,5,7,23-33]$ "educating and training" were considered in 14 studies. And, "support facilities" and "emergency medicines" were considered in 13 studies; while, "infrastructures" were considered in 8 studies (Table 3 ).

\section{Domains}

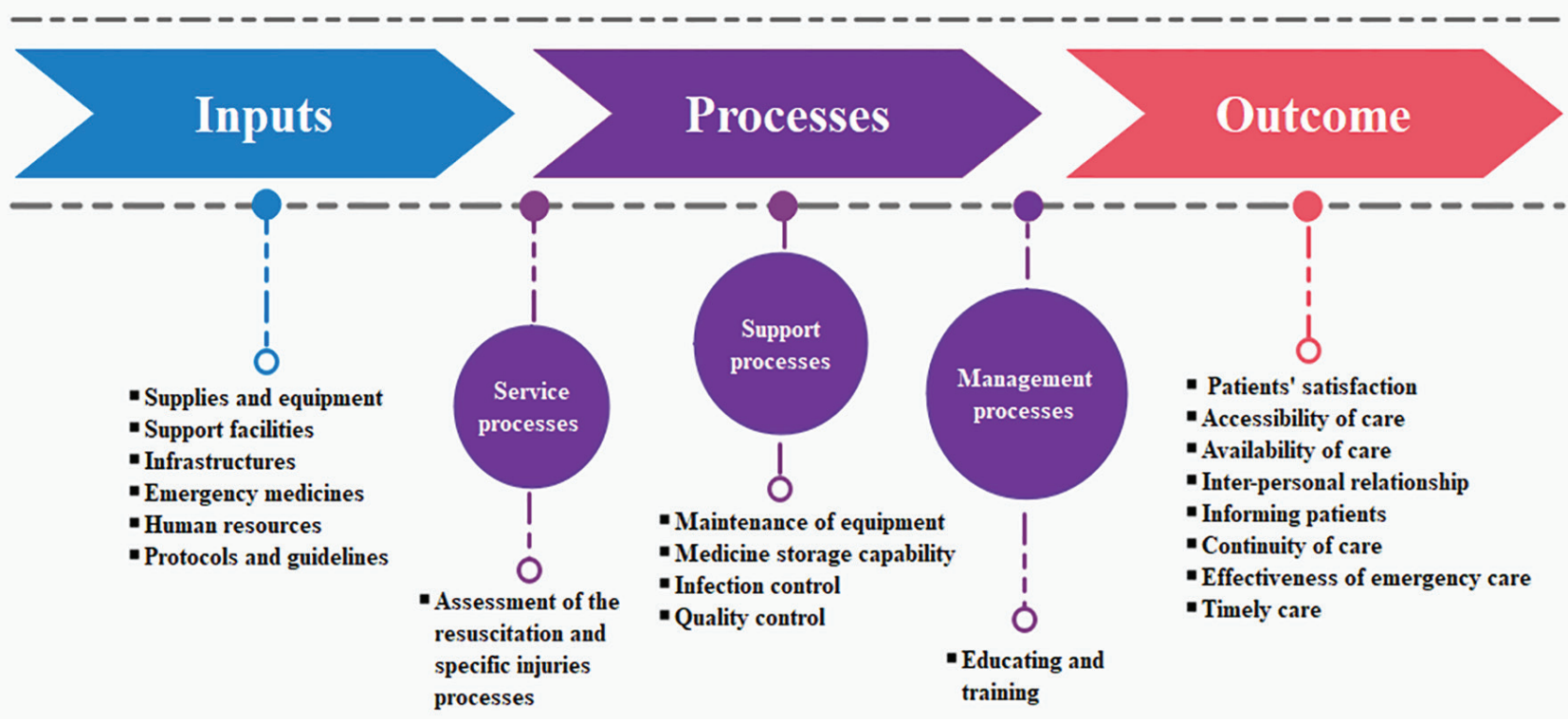

Fig. 2. Conceptual framework to assess non-hospital health Centers' preparedness to provide primary emergency care 
Table 3. Assessment Domains Considered in Preparedness to Provide Praimary Emergency Care

Author/Refrence

\begin{tabular}{|c|c|c|c|c|c|c|c|c|c|c|c|c|c|c|c|c|c|}
\hline Assessment & t Domains & 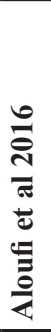 & 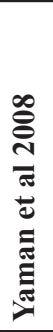 & 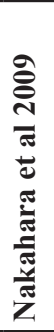 & 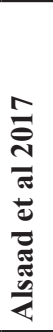 & 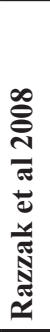 & 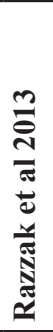 & 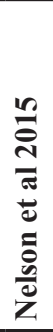 & 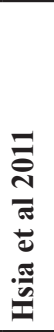 & 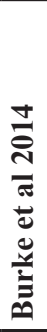 & 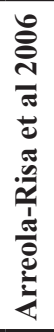 & 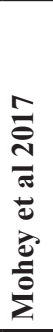 & 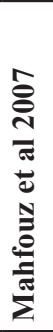 & 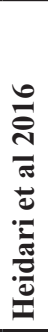 & 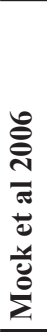 & 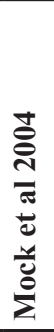 & Z \\
\hline \multirow[t]{6}{*}{ Inputs } & $\begin{array}{l}\text { Medical supplies } \\
\text { and equipment }\end{array}$ & $\sqrt{ }$ & $\sqrt{ }$ & $\sqrt{ }$ & $\sqrt{ }$ & $\sqrt{ }$ & $\sqrt{ }$ & $\sqrt{ }$ & $\sqrt{ }$ & $\sqrt{ }$ & $\sqrt{ }$ & $\sqrt{ }$ & $\sqrt{ }$ & $\sqrt{ }$ & $\sqrt{ }$ & $\sqrt{ }$ & 15 \\
\hline & Support facilities & $\sqrt{ }$ & $\sqrt{ }$ & $=$ & $\sqrt{ }$ & $\sqrt{ }$ & $\sqrt{ }$ & $\sqrt{ }$ & $\sqrt{ }$ & $\sqrt{ }$ & $\sqrt{ }$ & $\sqrt{ }$ & $\sqrt{ }$ & $=$ & $\sqrt{ }$ & $\sqrt{ }$ & 13 \\
\hline & Infrastructures & - & - & - & $\sqrt{ }$ & $\sqrt{ }$ & $=$ & $=$ & $\sqrt{ }$ & $=$ & $=$ & $\sqrt{ }$ & $\sqrt{ }$ & $\sqrt{ }$ & $\sqrt{ }$ & $\sqrt{ }$ & 8 \\
\hline & $\begin{array}{l}\text { Emergency } \\
\text { medicines }\end{array}$ & $\sqrt{ }$ & $\sqrt{ }$ & - & $\sqrt{ }$ & $\sqrt{ }$ & $\sqrt{ }$ & $\sqrt{ }$ & $\sqrt{ }$ & $\sqrt{ }$ & - & $\sqrt{ }$ & $\sqrt{ }$ & $\sqrt{ }$ & $\sqrt{ }$ & $\sqrt{ }$ & 13 \\
\hline & Human resources & $\sqrt{ }$ & $\sqrt{ }$ & $\sqrt{ }$ & $\sqrt{ }$ & $\sqrt{ }$ & $\sqrt{ }$ & $\sqrt{ }$ & $\sqrt{ }$ & $\sqrt{ }$ & $\sqrt{ }$ & $\sqrt{ }$ & $\sqrt{ }$ & $\sqrt{ }$ & $\sqrt{ }$ & $\sqrt{ }$ & 15 \\
\hline & $\begin{array}{l}\text { Protocol \& } \\
\text { Guideline }\end{array}$ & $=$ & $=$ & $=$ & $=$ & $=$ & $\sqrt{ }$ & $\sqrt{ }$ & $=$ & $=$ & $=$ & $\sqrt{ }$ & $=$ & $=$ & $=$ & $=$ & 3 \\
\hline \multirow[t]{6}{*}{ Processes } & $\begin{array}{l}\text { Assessment of } \\
\text { rescucitationand } \\
\text { specific injuries } \\
\text { processes }\end{array}$ & $\sqrt{ }$ & $\sqrt{ }$ & $\sqrt{ }$ & $=$ & $=$ & $\sqrt{ }$ & $=$ & $=$ & $=$ & $=$ & $=$ & $=$ & $=$ & $\sqrt{ }$ & $\sqrt{ }$ & 6 \\
\hline & $\begin{array}{l}\text { Maintenance of } \\
\text { equipment }\end{array}$ & - & - & - & - & - & - & - & $\sqrt{ }$ & - & - & - & - & - & - & - & 1 \\
\hline & $\begin{array}{l}\text { Medicine storage } \\
\text { capability }\end{array}$ & - & - & - & - & - & - & - & $\sqrt{ }$ & - & - & - & - & - & - & - & 1 \\
\hline & Infection control & - & - & - & - & - & - & - & $\sqrt{ }$ & - & - & - & - & - & - & - & 1 \\
\hline & Quality control & - & - & - & - & - & - & - & $\sqrt{ }$ & - & - & - & - & - & - & - & 1 \\
\hline & $\begin{array}{l}\text { Educating and } \\
\text { training }\end{array}$ & $\sqrt{ }$ & $\sqrt{ }$ & $\sqrt{ }$ & $\sqrt{ }$ & $\sqrt{ }$ & $\sqrt{ }$ & $\sqrt{ }$ & $\sqrt{ }$ & $=$ & $\sqrt{ }$ & $\sqrt{ }$ & $\sqrt{ }$ & $\sqrt{ }$ & $\sqrt{ }$ & $\sqrt{ }$ & 14 \\
\hline Outcome & $\begin{array}{l}\text { Patients' } \\
\text { satisfaction }\end{array}$ & $\sqrt{ }$ & $=$ & $=$ & $=$ & $=$ & $=$ & $=$ & $=$ & $=$ & $=$ & $\sqrt{ }$ & $\sqrt{ }$ & $=$ & $=$ & $=$ & 3 \\
\hline
\end{tabular}

\section{Discussion}

In this systematic review, domains, subdomains and items related to assessing preparedness of nonhospital centers for providing primary emergency care were identified. Then, they were structured based on Donabedian's triple model and a conceptual framework, which could be used to appraise preparedness of non-hospital centers when facing emergency cases, was presented.

The most important domain regarding assessing preparedness of non-hospital centers was "medical supplies and equipment". The effective domain that is required in assessing preparedness of nonhospital centers to provide primary emergency care to patients was "medical supplies and equipment". If any lack in this domain disturbs timely and effective treatment of patients, it may lead to some irrecoverable damages [34]. Medical supplies and equipment should be provided in accordance with real requirements of the centers and also qualified human forces. Moreover, they should have high quality, be adequate, and be supplied from reliable resources in determined time and for a reasonable price [35].

The most fundamental section of health care is human forces. They should possess a strong theoretical and practical base in intensive care since, in most of the cases, reaction of the patient or the injured individual concerns the manner in which the personnel treat the patient or his family [36]. Hence, efficient and sophisticated human forces should be employed to provide emergency care. And, saving the patient's life in the shortest possible time should be of the priorities of any measure and care [37]. When appraising the domain concerning infrastructures, noticing physical space for addressing the needs of emergency patients is essential. It is a space that can provide essential facilities to perform all the services in the shortest and fastest possible time with optimum efficiency. Such a space should be in line with the criterion of non-hospital health centers activities [1].

Assessment of the process associated with resuscitation and specific injuries is the most important domain in the section allocated to process in Donabedian model [5, 7, 24, 25, 32, 33]. Noticing two items in this domain is essential. One of the items is knowledge and skill of the personnel and the other is basic emergency equipment to provide emergency care. Basic knowledge/skills of the personnel in the processes like chin lift or jaw thrust, three-way dressing, and pelvic wrapping, are effective in saving injured patients particularly in areas remote [25]. The 
personnel who lack adequate skill and knowledge may fail to use available resources effectively [5].

Educating and training personnel has two items one of which is training courses for basic and advanced life supports and the other is continuing medical education. Training courses have a lot to do with remarkable increase in knowledge and skills of service providers. The point was considered in the studies of Razzak, Alsaad (2017), Nakahara (2009), Yaman (2008), Aloufi (2016), Mahfouz (2007) and Mock, Mohey (2017), Arreola (2006), Nelson (2015), (2008,2013), (2004, 2006). Training course include BLS, ATLS and etc. A trial in Trinidad \& Tobago in a local hospital in Somalia showed a 50\% decrease in deaths resulting from trauma injury after the physicians participated in Advanced Trauma Life Supports course [38]. Similarly, Hesam et al. also showed that training rescuers in Advanced Trauma Life Support skills in Iraq and Cambodia has decreased deaths resulting from local traumas from $22.6 \%$ to $13.7 \%$ over last two years [39].

Continuous education and training not only is important for the safety patients but also is necessary to motivate service providers, particularly those working in rural areas. On the other hand, it is required for developing some plans to improve accessibility of BLS. Education and training are in considered in the studies of Razzak $(2008,2013)$, Alsaad (2017), Heidari (2016), Hisa (2011), Aloufi (2016), Mahfouz (2007) and Mock (2004, 2006), Mohey (2017), Arreola (2006).

Assessing patient satisfaction plays a pivotal role in taking some measures to provide optimal emergency care and be more responsive to the needs of patients. It also proves that there is a need to improve health centers to provide highquality and effective care [40]. Further, it helps managers and policy makers to allocate resources and promote efficiency. Hence, policy makers and managers should have better understanding of what contributes to people's welfare [31]. The items concerning patient satisfaction include accessibility and availability of emergency care, interpersonal interaction, informing patients, continuity of care, comprehensive care, and efficient and timely care, which were considered in the studies of Aloufi (2016), Alsaad (2017), and Mohey (2017) [4, 24, 31].

Focus of the majority of studies was on input and processes since resource allocation structure in lowincome countries is often pretty poor. However, the outcome of patients is likely to be clear. Hence, lack of fundamental resources is a challenge that should be tackled. Nevertheless, we cannot deny the necessity of outcome in appraisals. In this systematic review, the available studies on assessing preparedness of non-hospital centers to provide primary emergency care were identified. This conceptual framework identifies elements that significantly affect the preparedness of non-hospital health centers to provide primary emergency care, so this framework may assist managers and researchers to take a comprehensive approach to assess these centers. However, it seems that to make such a conceptual framework valid, there is need for further studies so that precise and clear criteria can be identified so as to design flexible non-hospital centers for providing primary emergency care. Nevertheless, even results of this systematic review can be employed to develop a standard and valid tool.

\section{Study Strengths and Limitations}

Many aspects of this review imply that valid results have been reported. First, the research question was raised and then a comprehensive search strategy was devised to identify studies which focused on the non-hospital health centers and emergency care. Not only were electronic databases searched, but gray literature was also conducted. In addition, our literature review includes other search strategies, such as manually searching reference lists, and citations of the studies included and searching ResearchGate social network. No time limit was applied and the search was performed through a broad list of search terms to maximize research sensitivity. All the steps of systematic review, namely screening, quality control, and data extraction, were independently performed by two researchers.

As far as we know, this systematic literature review is the first attempt to identify prevalent domains related to the preparedness of non-hospital health centers to provide primary emergency care. This systematic review had very few limitations. A limitation was that the review was done only for studies published in English or Persian. Therefore, studies published in other languages may be left out of the analysis. The most important limitation was that very few studies have been done in the world.

In conclusion, a conceptual framework was constructed that identifies elements that significantly affect the preparedness of these centers. This framework may assist managers to take a comprehensive approach to assess these centers.

\section{Acknowledgment}

This study is part of an MSc dissertation which was financially supported by Tabriz University of Medical Sciences (Grant number: 59656).

Conflicts of Interest: None declared. 


\section{References}

1. Heidari A, Kabir MJ, Jafari N, Gashti AB, Pourabasi MA, Honarvar MR, et al. Assessment of human and physical resources in health houses and healthcare centers providing emergency services: a study in Golestan province. Health in the field. 2016;3(4):1-8.

2. Tabrizi JS, Amir Behghadami M, Saadati M, Söderhamn U. Selfcare Ability of Older People Living in Urban Areas of Northwestern Iran. Iran $J$ Public Health. 2018;47(12):1899-1905.

3. Montalto M. Outside the hospital: the delivery of healthcare in nonhospital settings. Public Health Assoc Australia Inc Po Box 319, Curtin, ACT 2600, Australia; 2009.

4. Mahfouz A, Abdel Moneim I, Khan M, Daffalla A, Diab M, El Gamal M, et al. Primary health care emergency services in Abha district of southwestern Saudi Arabia. East Mediterr Health J. 2007;13(1):103-12.

5. Razzak JA, Baqir SM, Khan UR, Heller D, Bhatti J, Hyder AA. Emergency and trauma care in Pakistan: a cross-sectional study of healthcare levels. Emerg Med J. 2013;32(3):207-13.

6. Almeida AOd, Araújo IEM, Dalri MCB, Araujo S. Theoretical knowledge of nurses working in nonhospital urgent and emergency care units concerning cardiopulmonary arrest and resuscitation. Rev Lat Am Enfermagem. 2011;19(2):261-8.

7. Yaman H. Preparedness of Primary Healthcare Centers for Critical Emergency Situations in Southwest Turkey. Prehosp Disaster Med. 2008;23(4):342-5.

8. Bosch $X$, Escoda O, Nicolás D, Coloma E, Fernández S, Coca A, et al. Primary care referrals of patients with potentially serious diseases to the emergency department or a quick diagnosis unit: a cross-sectional retrospective study. BMC Fam Pract. 2014;15(1):75.

9. Siddiqui S, Ogbeide DO. Utilization of emergency services in a community hospital. Saudi Med J. 2002;23(1):69-72.

10. Johnston CL, Coulthard MG, Schluter PJ, Dick M-LB. Medical emergencies in general practice in south-east Queensland: prevalence and practice preparedness. Med J Aust. 2001;175(2):99-103.

11. Toback SL. Medical emergency preparedness in office practice. $\mathrm{Am}$ Fam Physician. 2007;75(11):1679-84.

12. Fuchs S, Jaffe DM, Christoffel KK. Pediatric emergencies in office practices: prevalence and office preparedness. Pediatrics. 1989;83(6):931-9.

13. Jahani M, Rastgar A, Hosseinipour M, Bijani A. Structure of space, personnel and equipment of hospital emergency department of Babol university of medical sciences based on national standards (2007). J Babol Univ Med Sci. 2009;10(6):73-9.

14. Liberati A, Altman DG, Tetzlaff J, Mulrow C, Gøtzsche PC, Ioannidis JP, et al. The PRISMA statement for reporting systematic reviews and meta-analyses of studies that evaluate health care interventions: explanation and elaboration. PLoS Med. 2009;6(7):e1000100.

15. Arab-Zozani M, Pezeshki MZ, Khodayari-Zarnaq R, Janati A. Medical overuse in the Iranian healthcare system: a systematic review protocol. BMJ Open. 2018;8(4):e020355.

16. Amir Behghadami M, Janati A. A critical appraisal of the review study to improve its reporting quality. Iran $J$ Nurs Midwifery Res. 2019;24(4).

17. Hong QN, Pluye $P$, Fàbregues $S$, Bartlett G, Boardman F, Cargo M, et al. Mixed methods appraisal tool (MMAT), version 2018. IC Canadian Intellectual Property Office, Industry Canada. 2018.

18. O'Cathain A. Assessing the quality of mixed methods research: Toward a comprehensive framework. Handbook of mixed methods in social and behavioral research. 2010;531:555.

19. Crowe M, Sheppard L. A review of critical appraisal tools show they lack rigor: alternative tool structure is proposed. $J$ Clin Epidemiol. 2011;64(1):79-89.

20. Hsieh H-F, Shannon SE. Three approaches to qualitative content analysis. Qual Health Res. 2005;15(9):1277-88.

21. Potter WJ, Levine-Donnerstein D. Rethinking validity and reliability in content analysis; 1999. P. 258-84.

22. Donabedian A. An introduction to quality assurance in health care: Oxford University Press; 2002.

23. Hsia RY, Mbembati NA, Macfarlane S, Kruk ME. Access to emergency and surgical care in sub-Saharan Africa: the infrastructure gap. Health Policy Plan. 2012;27(3):234-44.

24. Aloufi MA, Bakarman MA. Barriers Facing Primary Health Care Physicians When Dealing with Emergency Cases in Jeddah, Saudi Arabia. Glob J Health Sci. 2016;8(8): 54248.
25. Nakahara $S$, Saint $S$, Sann $S$, Phy R, Ichikawa M, Kimura A, et al. Evaluation of trauma care resources in health centers and referral hospitals in Cambodia. World J Surg. 2009;33(4):874-85.

26. Alsaad SS, Abu-Grain SH, El-Kheir DY. Preparedness of Dammam primary health care centers to deal with emergency cases. J Family Community Med. 2017;24(3):181-8.

27. Razzak JA, Hyder AA, Akhtar T, Khan M, Khan UR. Assessing emergency medical care in low income countries: a pilot study from Pakistan. $B M C$ Emerg Med. 2008;8(1):8.

28. Nelson BD, Ahn R, Markovchick V, Hines RA, Lauretti AR, Lauretti JR, et al. Emergency care and referral among isolated island populations in the bahamas. J Emerg Med. 2015;48(3):356-65.

29. Burke TF, Hines R, Ahn R, Walters $\mathrm{M}$, Young D, Anderson RE, et al. Emergency and urgent care capacity in a resource-limited setting: an assessment of health facilities in western Kenya. BMJ Open. 2014;4(9):e006132.

30. Arreola-Risa C, Mock C, Vega Rivera F, Romero Hicks E, Guzmán Solana F, Porras Ramírez G, et al. Evaluating trauma care capabilities in Mexico with the World Health Organization's Guidelines for Essential Trauma Care publication. Rev Panam Salud Publica. 2006;19(2):94-103.

31. Mohey A, Faris Al azmi S. Primary Healthcare Emergency Services in Alexandria, Egypt 2016. Qual Prim Care. 2017;25(5):303-15.

32. Mock C, Nguyen S, Quansah R, Arreola-Risa C, Viradia R, Joshipura M. Evaluation of trauma care capabilities in four countries using the WHO-IATSIC Guidelines for Essential Trauma Care. World J Surg. 2006;30(6):946-56.

33. Mock C. Guidelines for essential trauma care: World Health Organization; 2004..

34. Rahmani H, Arab M, Akbari F, Zeraati H. Structure, process and performance of the emergency unit of teaching hospitals of Tehran University of Medical Sciences. $J$ Sch Public Health Inst Public Health Res. 2006;4(4):13-22.

35. Amerioun A, Toufighi S, Zaboli R. Assessing the medical equipment maintenance management at selected hospitals affiliated with the medical sciences universities in Tehran;(2003-2005). J Health Adm. 2006;9(23):17-24. 
36. Malek ML, Haghpanah S, Moravej H, Sharifi M. The effect of intervention on patient's satisfaction in emergency departments of the hospitals affiliated to shiraz university of medical sciences. J Jahrom Univ Medl Sci. 2010;7(3):52-61.

37. Bahadori M, Mirhashemi S, Panahi F, Sh T, Zaboli R, Rezaee M. Structure, process and practices of the emergency unit of hospitals of baqyatallah
University of Medical Sciences. $J$ Mil Med. 2008;9(4):257-63.

38. Ali J, Adam R, Butler AK, Chang $\mathrm{H}$, Howard M, Gonsalves D, et al. Trauma outcome improves following the advanced trauma life support program in a developing country. $J$ Trauma. 1993;34(6):890-8; discussion 8-9.

39. Husum $H$, Gilbert M, Wisborg $T$, Van Heng Y, Murad M. Rural prehospital trauma systems improve trauma outcome in low-income countries: a prospective study from North Iraq and Cambodia. J Trauma Acute Care Surg. 2003;54(6):1188-96.

40. Glick P. How reliable are surveys of client satisfaction with healthcare services? Evidence from matched facility and household data in Madagascar. Soc Sci Med. 2009;68(2):368-79. and share. Bulletin of Emergency And Trauma articles are published under a Creative Commons license (CC-BY-NC). 